\title{
Probing Membrane Viscosity and Inter-leaflet Friction of Supported Lipid Bilayers by Tracking Electrostatically Adsorbed, Nano-Sized Vesicles
}

\author{
Seyed R. Tabaeit, ${ }^{\dagger, t,}$ Jurriaan J. J. Gillissen ${ }^{+,+,}$, and Nam-Joon Cho ${ }^{*,+,+, \xi}$ \\ $\dagger$ School of Materials Science and Engineering, Nanyang Technological University, 50 Nanyang \\ Avenue 639798, Singapore \\ ॠCentre for Biomimetic Sensor Science, Nanyang Technological University, 50 Nanyang Drive \\ 637553, Singapore \\ ${ }^{\S}$ School of Chemical and Biomedical Engineering, Nanyang Technological University, 62 Nanyang \\ Drive 637459, Singapore
}

Seyed Tabaei and Jurriaan Gillissen contributed equally to this work.

Keywords: (supported lipid bilayers, membrane viscosity, inter-leaflet friction, lipid vesicles, single particle tracking)

Investigating the nanorheology of fluid membranes is of both fundamental and practical importance for understanding the dynamics of biomembranes. Particle tracking is used to measure the diffusional motion of nano-sized $(\sim 100 \mathrm{~nm})$, unilamellar, lipid vesicles that are electrostatically adsorbed onto a solid supported lipid bilayer. We find that the motion of the membrane-adhering vesicles is Brownian and depends inversely on the vesicle size, but, surprisingly, is insensitive to the vesicle surface charge. The measured diffusivity agrees well with the Evans - Sackmann model [J. Fluid Mech. 1988, 194, 553-561] for the diffusion of inclusions in supported, fluidic membranes. The agreement implies that the vesicle motion is coupled to that of a nanoscopic lipid cluster in the upper leaflet, which slides over the lower leaflet. The diffusivity of the membrane adhering vesicles is therefore predominantly governed by the inter-leaflet friction coefficient, while the diffusivity of single lipids is mainly governed by the membrane viscosity. 
Consequently combined with fluorescence recovery after photobleaching analysis, we determine the inter-leaflet friction coefficient and the membrane viscosity, by applying the Evans - Sackmann model to the measured diffusivity of membrane adhering vesicles and that of supported membrane lipids. This approach provides a simple alternative to existing methods for measuring the interleaflet friction coefficient and the membrane viscosity.

\section{Introduction}

Fluidity is a fundamental property of biological membranes, which is crucial for cellular function. ${ }^{[1 \text {, }}$ 2] The corresponding lateral mobility of lipids and membrane proteins facilitates their dynamic rearrangements and spatial distribution, which are essential for a range of membrane-dependent processes, such as protein clustering during signaling, transport and cell-cell interaction. ${ }^{[3-6]}$ Owing to the relevance for biological function, many research efforts are directed towards characterizing the fluid mechanical properties of phospholipid bilayers. The basic fluid mechanical property of the lipid bilayer is the membrane viscosity. Measuring this quantity is difficult however, and various techniques have been developed in the literature. Most methods rely on measuring the diffusivity of tracer particles, embedded in or adhering to the membrane, and invoking a fluid mechanics model to translate the particle diffusivity to the membrane viscosity, where similar as in a three dimensional fluid, the particle diffusivity in a two dimensional fluidic membrane is nearly inversely proportional to the viscosity of the membrane; see for instance the Evans - Sackmann model [Eq. (1)] below. ${ }^{[7]}$ Following this approach, the diffusivity of membrane lipids or membrane proteins have widely been measured using fluorescence recovery after photo-bleaching, ${ }^{[8-10]}$ or fluorescence correlation spectroscopy. ${ }^{[11,12]}$ Translating diffusivity to viscosity however, using continuum fluid mechanics models, generally works better for larger particles, than individual molecules. Therefore, in an attempt to accurately determine the viscosity of lipid bilayers, single particle tracking has been used to measure the diffusivity of large membrane inclusions, such as phase-separated lipid 
domains ${ }^{[13-15]}$ or macroscopic particles, that are externally bound to the membrane. ${ }^{[16-19]}$ When translating the diffusivity of membrane adhering particles to the membrane viscosity, it has been assumed that the particles are associated to a disk of lipids, whose diffusivity follows the Saffman Delbrück model ${ }^{[20]}$ for membrane inclusions. ${ }^{[18,19]}$

In the present work, we apply a similar approach as adopted by Hormel et al. ${ }^{[19]}$, to determine the fluid mechanical properties of phospholipid bilayers by measuring the diffusivity of nano-sized ( $\sim 100 \mathrm{~nm})$, unilamellar vesicles (SUVs) adhering to a supported lipid bilayer (SLB). The SLB is a robust platform which mimics the basic architecture of biological membranes and preserves their fundamental characteristics such as bilayer thickness and two-dimensional fluidity. It is also widely used for studying the biophysical properties of phospholipid bilayers. ${ }^{[21-23]}$ In our experimental design, the SUVs are associated to the supported bilayer using a weak electrostatic force, as opposed to molecular tethers, which were used in most previous works to adhere colloidal-sized objects to membranes; see e.g. Refs.. ${ }^{[16,19]}$. We focus on the diffusion of SUVs on SLBs, which is different as on free-standing bilayers, ${ }^{[19]}$ due to the friction between the membrane and the solid support. Evans and Sackmann ${ }^{[7]}$ modeled this friction by introducing a phenomenological friction coefficient into the Saffman - Delbrück model. ${ }^{[20]}$ Assuming that the diffusivity of our membrane adhering SUVs induces slippage between the two leaflets of the SLB, we will determine the interleaflet friction coefficient $b$ by measuring the diffusivity of the membrane-adhering SUVs.

In the literature, there is a wide range of experimentally measured values for $b\left(10^{6}-10^{9} \mathrm{~kg} \mathrm{~m}^{-2}\right.$ $\mathrm{s}^{-1}$ ), suggesting that measuring $b$ is far from straightforward and highly sensitive to compositional details. Techniques to measure $b$ in free-standing membranes are based on induced leaflet slippage by forcing the bilayer through a region of high curvature,${ }^{[24-26]}$ thermal shape fluctuations, ${ }^{[27,28]}$ or chemically induced shape instabilities. ${ }^{[29]}$ The analysis involved in interpreting these measurements is difficult however. For supported lipid membranes, on the other hand, determining $b$ may be less 
involved. For instance Merkel et al. monitored the diffusion of lipids in the upper leaflet, while the lower leaflet was covalently bound onto the solid support. ${ }^{[9]}$ This method assumes applicability of the Evans - Sackmann model to describe the dynamics of individual membrane lipids. Jönsson and Beech determined $b$ by measuring the shear-induced drift velocity of the upper leaflet, sliding over the solid-supported lower leaflet. ${ }^{[10]}$ These authors validated the assumption of Merkel et al. ${ }^{[9]}$, regarding the applicability of the Evans - Sackmann model to describe diffusional motion of individual lipids. They furthermore showed that the friction coefficient between the bilayer and the solid support exceeds the friction coefficient between the leaflets, presumably due to an exceedingly large viscosity of the water molecules in the nanoscopic layer between the membrane and the solid surface. In this work we use this observation and assume a relatively immobile lower leaflet. We furthermore assume that the SUVs are rigidly bound to nanoscopic domains in the upper membrane leaflet. Under these assumptions, the motion of these lipid domains, which are probed via the associated, fluorescently labeled SUVs, correspond to substantial inter-leaflet slippage, which allows the determination of the inter-leaflet friction coefficient $b$. We will validate these assumptions by showing good agreement between the resulting $b$ and literature values.

\section{Results and Discussion}

Positively charged, fluorescently labeled, solid supported lipid bilayers (SLBs) were formed on the glass wall of a fluidic channel, by employing the vesicle fusion method. ${ }^{[30]}$ A fluorescence microscopy image of the bilayer is provided in Figure 1a. After SLB formation fluorescently labeled, negatively charged, small unilamellar vesicles (SUVs) were injected into the fluidic channel, where they electrostatically adhere and diffuse on the SLB (see Supporting Video S1). The fluidity of the supported bilayer was examined by fluorescence recovery after photobleaching (FRAP) as shown in Figures 1b-d. The diffusivity of the membrane lipids was found to be $2.5 \pm$ $0.5 \mu \mathrm{m}^{2} \mathrm{~s}^{-1}$, which is within the expected range for fluid planar bilayers. ${ }^{[31-33]}$ In addition Figures 
1b-d show the membrane adhering vesicles, which appear as bright spots. The insets of Figures 1c and 1d show a reconstructed particle trajectory at $t=40 \mathrm{~s}$ and at $t=120 \mathrm{~s}$, respectively. Over this time interval, a typical SUV diffuses over a distance of around $5 \mu \mathrm{m}$, while the membrane lipids diffuse over a larger distance of around $20 \mu \mathrm{m}$ (diameter of bleached spot). This illustrates the slow diffusion of membrane adhering SUVs, as compared to the rapid diffusion of individual membrane lipids.

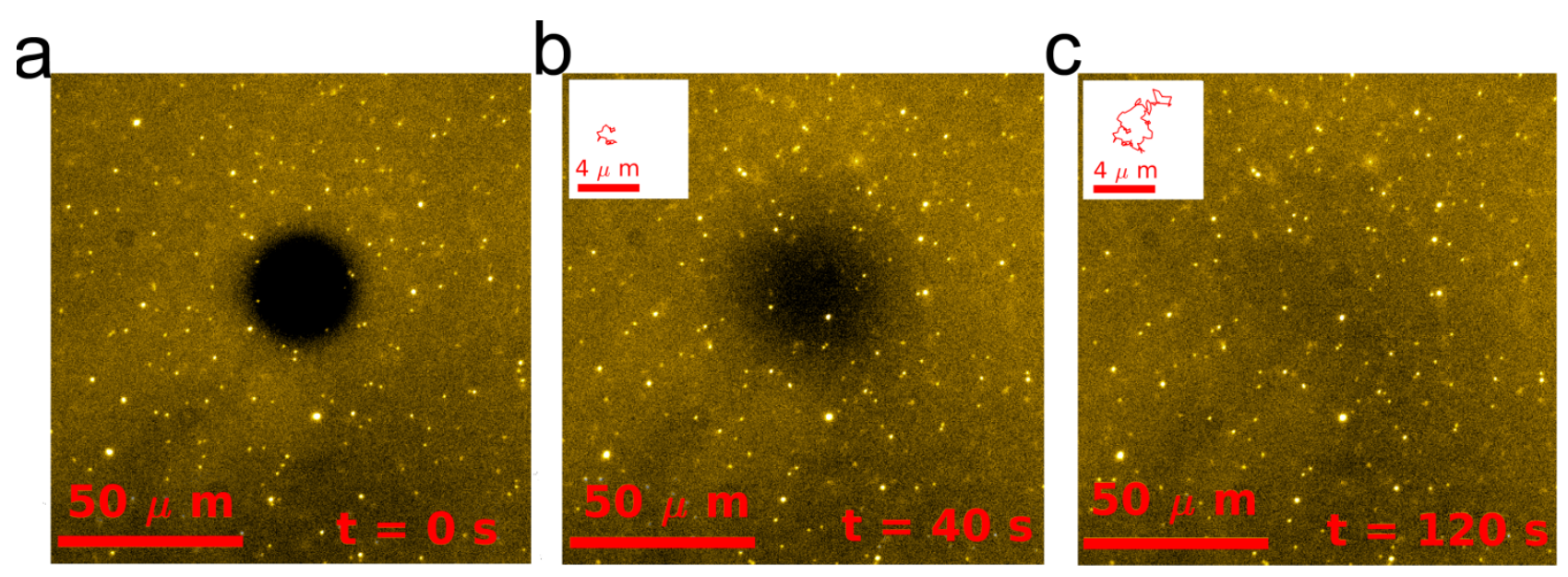

Figure 1. Fluorescence micrographs of small unilamellar vesicles (SUVs) diffusing on a solid supported lipid bilayer (SLB). A fluorescence microscopy image of the SLB without SUVs (a) and three fluorescence microscopy images of the SLB with SUVs, directly after photobleaching (b), $40 \mathrm{~s}$ after photobleaching (c) and $120 \mathrm{~s}$ after photobleaching (d). Membrane adhering SUVs are visible as bright spots. The insets of (c) and (d) show a reconstructed particle trajectory at $t=40 \mathrm{~s}$ and $t=120 \mathrm{~s}$, respectively. During $120 \mathrm{~s}$ the corresponding SUV diffuses 5 $\mu \mathrm{m}$, while the membrane lipids diffuse $20 \mu \mathrm{m}$ (diameter of bleached region).

After reconstructing the SUV trajectories, the statistics of the SUV displacements (the probability of the distance $r$ travelled in a certain amount of time $t$ ) were analyzed, by treating the horizontal and vertical displacements, separately. Figure 2a shows for various time intervals the resulting probability density function $G(r, t)$ of the vesicle displacement, normalized by twice the square root of time. The distribution $G(r, t)$ is shown on a logarithmic $y$-axis, where data for different times collapse onto a straight line, which implies that the displacements are Gaussian 
distributed: $G=\exp \left(-r^{2} / 4 D t\right) /(4 \pi D t)^{1 / 2}$.
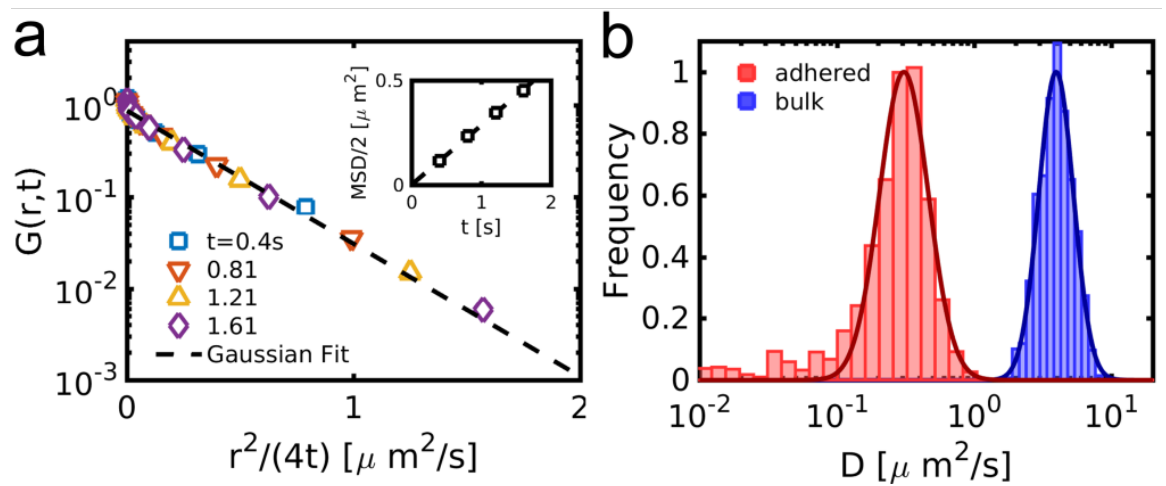

Figure 2. Mobility analysis of membrane adhering vesicles. (a) The SUV displacement probability $G(r, t)$ on a logarithmic $y$-axis, as a function of the vesicle displacement $r$ scaled with the square root of time $t$. The data for different times collapse on a straight line, which indicates that the displacements are Gaussian distributed. The inset shows the mean squared displacement as a function of the elapsed time. The data follow a straight line, which indicates that the motion is Brownian. (b) Vesicle diffusivity histogram for adhering vesicles (red) and bulk vesicles (blue; measured with NTA).

To check whether the motion of the membrane-adhering SUVs is Brownian, the mean squared displacement (MSD) between any two points on each trajectory was computed and plotted as a function of the elapsed time (see the inset of Figure 2a). The data shows that the $\operatorname{MSD}(t)$ is linear, which confirms that the motion is Brownian.

Next the MSD for each vesicle was computed, and its diffusivity $D$ was determined as the slope of the corresponding $\operatorname{MSD}(t)$ curve. Figure 2b shows the resulting diffusivity distribution for membrane-adhering SUVs (red bars) with a mean value of $0.28 \mu \mathrm{m}^{2} \mathrm{~s}^{-1}$, which is 17 times smaller than the diffusivity of vesicles from the same batch that are freely floating in the bulk $\left(4.8 \mu \mathrm{m}^{2} \mathrm{~s}^{-1}\right)$. The latter distribution (blue bars) was obtained from nanoparticle tracking analysis (NTA), which measures particle diffusivity in a three dimensional fluid, by tracking the random particle motions. NTA provides as output the particle size distribution, which is related to the particle diffusivity distribution via the Stokes - Einstein relation: $D=k_{\mathrm{B}} T / 6 \pi \eta a$, where $\eta$ is the viscosity of the fluid, $a$ 
is the radius of the particle and $k_{\mathrm{B}} T$ is the Boltzmann energy,. The smaller diffusivity for the membrane-adhering SUVs indicates a large friction between the SUVs and the SLB. The observed spread in the diffusivity of the membrane-adhering vesicles has several causes; e.g. inhomogeneities in the supported membrane, variations in the vesicle size, variations in the surface charge properties as well as statistical fluctuations due to the stochastic nature of the diffusion process itself.

Next we modeled the diffusivity of the membrane adhering SUVs using the Evans - Sackmann model for cylindrical particles embedded within a solid supported, fluidic membrane: ${ }^{[7]}$

$D=\frac{k_{B} T}{4 \pi \eta_{M}}\left(\frac{1}{2} \varepsilon^{2}+\frac{\varepsilon K_{1}(\varepsilon)}{K_{0}(\varepsilon)}\right)^{-1}$, where $\varepsilon=a_{C} \sqrt{\frac{b}{\eta_{M}}}$

In Eq. (1) $a_{\mathrm{C}}$ is the inclusion radius, $\eta_{\mathrm{M}}$ is the membrane viscosity, $K_{0}$ and $K_{1}$ are the zeroth and first order modified Bessel functions of the second kind, and $b$ is a phenomenological friction coefficient, to account for the presence of the solid support. As we will see below, in our system the presence of the solid support induces slippage between the lipids in the upper leaflet and lipids in the lower leaflet. Furthermore, since we apply Eq. (1) to describe diffusivity of (individual or clusters of) lipids in the upper leaflet, we interpret $b$ and $\eta_{M}$ in Eq. (1) as the inter-leaflet friction coefficient and the monolayer (half the bilayer) viscosity, respectively. The relative importance of these two material properties is determined by the dimensionless inclusion radius: $\varepsilon=a_{\mathrm{C}} / a^{*}$, where $a^{*}=\left(\eta_{\mathrm{M}} / 2 b\right)^{1 / 2}$ is a length scale, which is estimated as $a^{*} \approx 6 \mathrm{~nm}$, using the experimentally found values of $\eta_{\mathrm{M}} \approx 7 \times 10^{-10} \mathrm{~kg} \mathrm{~s}^{-1}$ (bilayer viscosity) and $b \approx 1 \times 10^{7} \mathrm{~kg} \mathrm{~m}^{-2} \mathrm{~s}^{-1}$ (see below). The length scale $a^{*}$ defines a transition in diffusional behavior from one that is dominated by the membrane viscosity to one that is dominated by the inter-leaflet friction. When $a_{\mathrm{C}}<<a^{*}(\varepsilon<<1)$ the diffusivity $D$ is governed by the membrane viscosity $\eta_{\mathrm{M}}$ as given by: $D \sim k_{\mathrm{B}} T / \eta_{\mathrm{M}}$, while when $a_{\mathrm{C}}>>$ $a^{*}(\varepsilon>>1)$ the diffusivity $D$ is dominated by the inter-leaflet friction coefficient $b$ as given by: $D \sim$ $k_{\mathrm{B}} T / b a_{\mathrm{c}}^{2}$, where we ignored the numerical and weak (logarithmic) factors in the expressions for these limiting cases. 
In order to use Eq. (1) to describe the diffusivity of membrane adhering SUVs, we interpret $a_{\mathrm{C}}$ as the radius of the contact area between the SUV and the SLB, which is modeled, as a circular region of the SLB that is within electrostatic range of the SUV, i.e. within one Debye length $\lambda$, as illustrated in Figure 3. In the Supporting Information we derive the following expression for the contact radius $a_{\mathrm{C}}$ of a sphere with radius $a$, which is valid in the limit: $a / \lambda>>1$ :

$a_{C}=\sqrt{2 a \lambda}$

It is noted that according to Eq. (2), the contact area and the corresponding diffusivity are insensitive to the membrane charge density, and we will verify this aspect below.

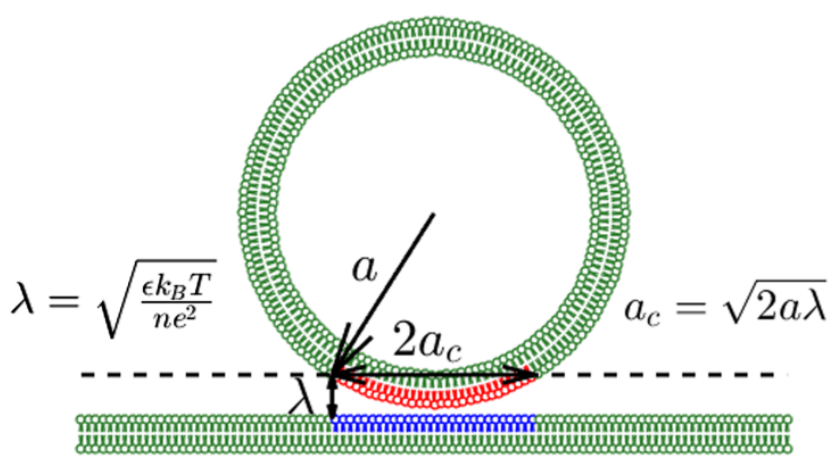

Figure 3. Schematic representation of a small unilamellar vesicle (SUV) that is electrostatically adhering to a supported lipid bilayer (SLB). A cluster of SUV lipids (red) is assumed to be electrostatically bound to a disk-shaped cluster of SLB lipids (blue). The radius of the disk is referred to as the contact radius $a_{\mathrm{C}}$, which is modeled as the geometric mean of the SUV diameter: $2 a$ and the Debye length $\lambda$, i.e.: $a_{\mathrm{C}}=(2 a \lambda)^{1 / 2}$. The Debye length $\lambda=\left(\varepsilon k_{\mathrm{B}} T / n e^{2}\right)^{1 / 2}$ depends on the Boltzmann energy $k_{\mathrm{B}} T$, the unit charge $e$, the electric permittivity of water $\varepsilon$, and the number of counterions per unit volume $n=2 \times 10^{3} N_{\mathrm{A}} c$, where $c$ is the $\mathrm{NaCl}$ molarity and $N_{\mathrm{A}}$ is Avogadro's constant.

In our system, the ionic strength is $150 \mathrm{mM}(\mathrm{NaCl})$ and the (mean) vesicle radius is: $a=53 \mathrm{~nm}$ (see Figure 4a), which gives for the Debye length: $\lambda=0.8 \mathrm{~nm}$ and for the contact radius: $a_{\mathrm{C}}=9 \mathrm{~nm}$, which is larger than the cross-over length: $a^{*} \approx 6 \mathrm{~nm}$ (see above), suggesting that the diffusivity of the membrane adhering SUVs is predominantly governed by the inter-leaflet-friction coefficient $b$. Using this estimate for the contact radius, we applied Eq. (1) to model the diffusivity of the 
adhering SUVs. To this end we realize that the disk of bound lipids resides within the upper leaflet of the SLB (blue lipids in Figure 3). Since the friction coefficient between the lower leaflet and the solid support has been shown to be much larger than the inter-leaflet friction coefficient, ${ }^{[10]}$ it is assumed that vesicle motion induces slippage between the moving lipid cluster in the upper leaflet and the relatively steady lower leaflet. Therefore, as mentioned above, the membrane viscosity $\eta_{\mathrm{M}}$ and the friction coefficient $b$ in Eq. (1) are interpreted as half the bilayer (monolayer) viscosity and the inter-leaflet friction coefficient.

We determined these material properties by applying Eq. (1) to the measured vesicle diffusivity: $D=0.28 \pm 0.14 \mu \mathrm{m}^{2} \mathrm{~s}^{-1}$ and $a_{\mathrm{C}}=9 \mathrm{~nm}$ as well as to the diffusivity of a single lipid in the SLB: $D=$ $2.5 \pm 0.5 \mu \mathrm{m}^{2} \mathrm{~s}^{-1}$ and $a_{\mathrm{C}}=0.45 \mathrm{~nm}$, where $a_{\mathrm{C}}$ represents the radius of a single lipid molecule. ${ }^{[9]}$ Although the applicability of continuum fluid mechanics models, such as Eq. (1), is questionable at the nanometer scale, this approach has been proven to provide reasonable results in similar situations; see e.g. Refs. ${ }^{[9,10,34]}$. Inserting these values into Eq. (1) and solving the resulted system of two equations, provided the inter-leaflet friction coefficient: $b=(1.1 \pm 0.6) \times 10^{7} \mathrm{~kg} \mathrm{~s}^{-1} \mathrm{~m}^{-2}$ and the bilayer viscosity (twice the monolayer viscosity): $2 \times \eta_{\mathrm{M}}=(7.0 \pm 3.5) \times 10^{-10} \mathrm{~kg} \mathrm{~s}^{-1}$, which are remarkably close to previously reported values: $b=2 \times 10^{7} \mathrm{~kg} \mathrm{~s}^{-1} \mathrm{~m}^{-2}$ and $2 \times \eta_{\mathrm{M}}=4 \times 10^{-10} \mathrm{~kg} \mathrm{~s}^{-1}{ }^{[10]}$ This agreement implies that the diffusivity of an electrostatically adhering SUV is equivalent to that of a membrane inclusion, whose size $a_{\mathrm{C}}$ is determined by the electrostatic coupling between the SUV and the underlying SLB. The contact radius $a_{\mathrm{C}}$ is assumed to be independent of the charge density in the opposing membranes, but modeled here as the geometric mean of the SUV diameter $2 a$ and the Debye length $\lambda$, as given by Eq. (2). We have performed a series of control experiments, to test the presumed relation between the contact radius $a_{\mathrm{C}}$ and three operational parameters, being the vesicle radius $a$, the Debye length $\lambda$ and the membrane charge density.

First the relation between the contact radius and the vesicle size was verified by comparing the 
diffusivity of vesicles that are produced by extrusion through membranes with a pore size of either $50 \mathrm{~nm}$ or $100 \mathrm{~nm}$. The radii of the corresponding vesicles are measured with dynamic light scattering (DLS), giving: $a=53 \pm 15$ and $87 \pm 28 \mathrm{~nm}$ and the resulting size distributions are provided in Figure 4a. According to Eqs. (1) and (2), an $87 \mathrm{~nm}$ vesicle has a 30\% smaller diffusivity than a $53 \mathrm{~nm}$ vesicle. This prediction agrees perfectly with the experimental data, presented in Figure $\mathbf{4 b}$, showing that the mean of the diffusivity distributions for the small and the large vesicles are $0.30 \mu \mathrm{m}^{2} \mathrm{~s}^{-1}$ and $0.21 \mu \mathrm{m}^{2} \mathrm{~s}^{-1}$, respectively. In Figure $4 \mathbf{c}$ we further verified the inverse relation between the diffusivity and the vesicle size [Eqs. (1) and (2)]. In that figure we plotted the measured diffusivity of individual vesicles against their size, where the vesicle size was estimated from the square root of the fluorescence emitted from an individual vesicle (see Supporting Information for detailed analysis). In this figure each dot corresponds to a single vesicle. The measured SUV diffusivity decreased with increasing vesicle size, which supports the validity of the modeled relation between the contact radius and the vesicle size [Eq. (2)].
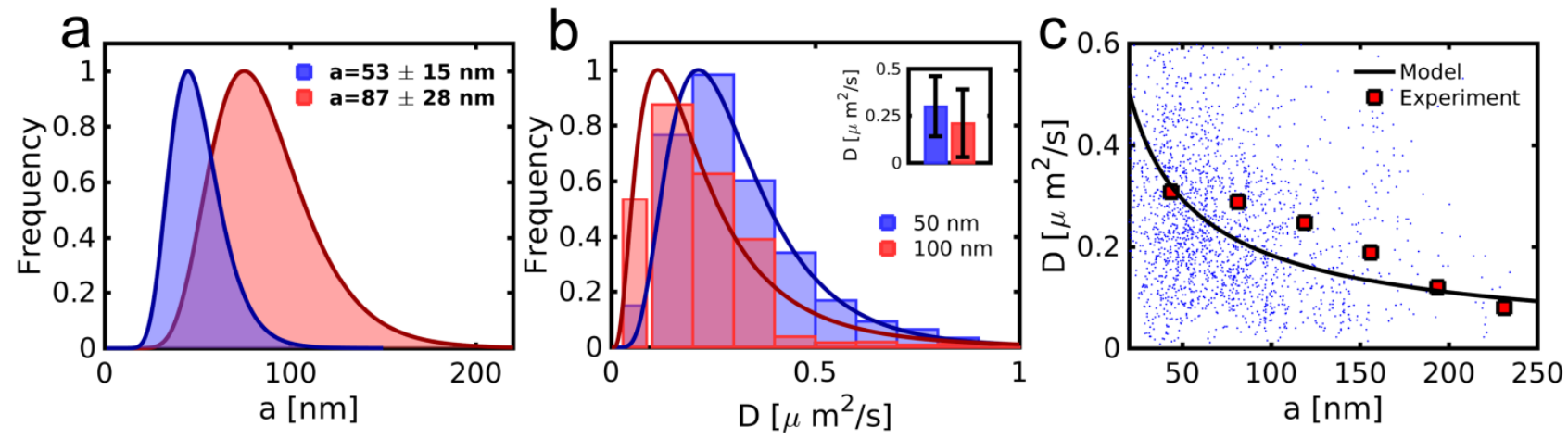

Figure 4. Effect of the size of a small unilammelar vesicle (SUVs) on its diffusivity on a supported lipid bilayer (SLB). (a) Size distribution measured by DLS of SUVs extruded through pores of $50 \mathrm{~nm}$ (blue) and $100 \mathrm{~nm}$ (red). (b) Diffusivity histogram for SUVs extruded through pores of $50 \mathrm{~nm}$ (blue) and $100 \mathrm{~nm}$ (red). The larger SUVs have a smaller diffusivity. (c) SUV diffusivity $D$ as a function of its radius $a$ (obtained from the square root of the emitted fluorescence per vesicle). Each dot corresponds to a single vesicle. The large red squares show the mean of the diffusivity as a function of the radius. The line corresponds to the model [Eqs. (1) and (2)], using $b=1.1 \times 10^{7} \mathrm{~kg} \mathrm{~s}^{-1} \mathrm{~m}^{-}$ ${ }^{2}, \eta_{\mathrm{M}}=1 / 2 \times 7.0 \times 10^{-10} \mathrm{~kg} \mathrm{~s}^{-1}$ and $\lambda=0.8 \mathrm{~nm}$. 
Next we verified the presumed relation between the contact radius and the Debye length. To this end, the diffusivity of membrane adhering SUVs was measured at two ionic strengths, i.e. $150 \mathrm{mM}$ and $10 \mathrm{mM} \mathrm{NaCl}$, which corresponds to a Debye length of 0.8 and $3.1 \mathrm{~nm}$, respectively. In this test we use vesicles containing 5\% charged lipids and extruded through $100 \mathrm{~nm}$ pores, which have an average size of $87 \mathrm{~nm}$ (see Figure 5a). According to Eq. (2) the contact radius $a_{\mathrm{C}}$ between the SUVs and the SLB at $150 \mathrm{mM}$ and $10 \mathrm{mM} \mathrm{NaCl}$ equals $12 \mathrm{~nm}$ and $23 \mathrm{~nm}$, respectively. Inserting these values together with $\eta_{\mathrm{M}}=1 / 2 \times 7 \times 10^{-10} \mathrm{~kg} \mathrm{~s}^{-1}$ and $b=1 \times 10^{7} \mathrm{~kg} \mathrm{~s}^{-1} \mathrm{~m}^{-2}$ into Eq. (1) we predicted a reduction of the SUV diffusivity from $0.20 \mu \mathrm{m}^{2} \mathrm{~s}^{-1}$ at $150 \mathrm{mM}$ ionic strength to 0.07 $\mu \mathrm{m}^{2} \mathrm{~s}^{-1}$ at $10 \mathrm{mM}$ ionic strength. These values are in excellent agreement with the experimental observation, that the ensemble averaged SUV diffusivity decreases from $0.21 \mu \mathrm{m}^{2} \mathrm{~s}^{-1}$ to $0.07 \mu \mathrm{m}^{2} \mathrm{~s}^{-1}$ upon reducing the ionic strength from $150 \mathrm{mM}$ to $10 \mathrm{mM} \mathrm{NaCl}$ (Figure 5b). This striking agreement further supports the assumptions underlying our modeling approach, e.g. rigidity of the cluster of bound membrane lipids and the spherical shape of the adhering SUVs.

Finally we tested the independence of the contact area on the vesicle charge density by measuring the diffusivity of SUVs with different charge densities ( $1 \mathrm{~mol} \%$ and $5 \mathrm{~mol} \%$ DOPS) but similar sizes (Figure 5a). The data in Figure 5c show that these SUVs have a similar diffusivity, which is compatible with Eq. (2), confirming that, within this parameter range, the contact radius $a_{\mathrm{C}}$, is insensitive to the SUV charge density, but instead is governed by the ionic strength, via the Debye length $\lambda$. 

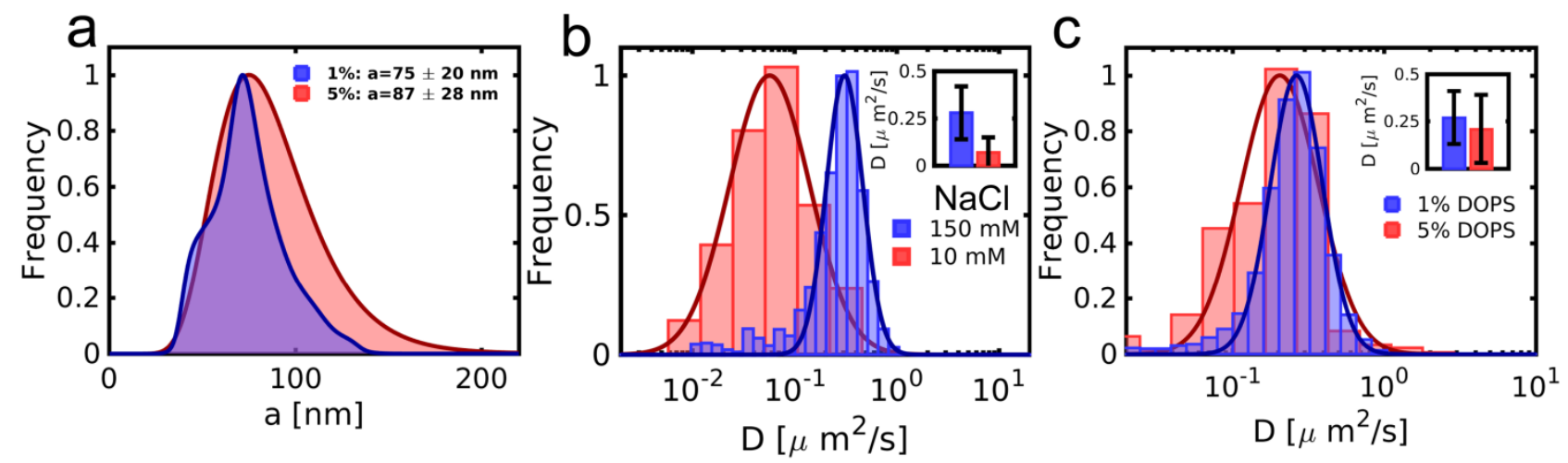

Figure 5. Effect of ionic strength and charge density of small unilamellar vesicles (SUVs) on their diffusivity on a supported bilayer (SLB). (a) Size distribution of DOPC vesicles prepared by extrusion through $100 \mathrm{~nm}$ polycarbonate filter containing $1 \mathrm{~mol} \%$ (blue) and $5 \mathrm{~mol} \%$ (red) charged lipids (DOPS). (b) Diffusivity of membrane adhering SUVs [DOPC:DOPS (95:5)] in a tris buffer containing $10 \mathrm{mM}$ (red) and $150 \mathrm{mM}$ (blue) $\mathrm{NaCl}$. The inset shows ensemble averaged diffusivity of the SUVs. (c) Diffusivity of DOPC vesicles prepared by extrusion through $100 \mathrm{~nm}$ polycarbonate filter containing $1 \mathrm{~mol} \%$ (blue) and $5 \mathrm{~mol} \%$ (red) charged lipids (DOPS). The inset shows ensemble averaged diffusivity of the SUVs.

The above control experiments support the validity of the contact area model [Eq. (2)], which, together with Eq. (1), allows extracting the membrane viscosity and the inter-leaflet friction coefficient from measuring the diffusivity of membrane lipids and membrane-adhering SUVs. Another assumption underlying our method is that the friction coefficient between the lower leaflet of the membrane and the solid support exceeds the friction coefficient between the upper leaflet and the lower leaflet, such that the SUV motion induces slippage between the upper and the lower leaflet. Validity of this assumption is supported by a remarkably good agreement between our measured value: $b=(1.1 \pm 0.6) \times 10^{7} \mathrm{~kg} \mathrm{~s}^{-1} \mathrm{~m}^{-2}$ and the value: $b=2 \times 10^{7} \mathrm{~kg} \mathrm{~s}^{-1} \mathrm{~m}^{-2}$ found by Jönsson and Beech, who applied a viscous shear stress to a supported lipid bilayer and showed a shear-induced motion in the upper leaflet while the lower leaflet was almost stationary. ${ }^{[10]}$

\section{Conclusion}

We have investigated the diffusional motions of small $(\sim 100 \mathrm{~nm})$ lipid vesicles, that are 
electrostatically adhering to a supported lipid bilayer. The measured diffusivity is interpreted in the light of the Evans -Sackmann model for diffusion of membrane inclusions. This modeling approach assumes, that the SUV is coupled to a disk of membrane lipids, that moves as a rigid object within the upper leaflet of the SLB, causing substantial inter-leaflet friction. The contact radius between the SUV and the SLB was modeled as the geometric mean of the SUV size and the Debye length. Applying the Evans - Sackmann model to experimental diffusivity data of both membrane lipids and membrane-adhering SUVs provides values for the membrane viscosity and the inter-leaflet friction coefficient in agreement with literature values.

\section{Experimental Section}

Materials. Zwitterionic 1,2-dioleoyl-sn-glycero-3-phosphocholine (DOPC), anionic 1,2-dioleoylsn-glycero-3-phospho-L-serine (DOPS), cationic sn-glycero-3-ethylphosphocholine (DOEPC) and fluorescently labeled 1,2-dioleoyl-sn-glycero-3-phosphoethanolamine-N-(lissamine rhodamine B sulfonyl) (rhodamine-PE) lipids were obtained from Avanti Polar Lipids.

Vesicle preparation. Small unilamellar vesicles were prepared by the extrusion method. Briefly, a chloroform solution of lipids was first dried by a stream of $\mathrm{N}_{2}$ and kept under vacuum for $3 \mathrm{~h}$. The dried film was then rehydrated with aqueous buffer $10 \mathrm{mM}$ Tris, $150 \mathrm{mM} \mathrm{NaCl}(\mathrm{pH}$ 7.5). After vortex mixing of the rehydrated solution, unilamellar vesicles of different size were made by a Mini Extruder (Avanti Polar Lipids) using a polycarbonate membrane with a pore size of $50 \mathrm{~nm}$ or 100 $\mathrm{nm}$. The vesicle size distribution was measured either by nanoparticle tracking analysis $(\mathrm{NTA})^{[35]}$ using a NanoSight LM10 instrument (NanoSight, Amesbury, UK) or by dynamic light scattering (Malvern Instruments).

Supported Bilayer Fluidity. The fluidity of the bilayer was investigated by measuring the fluorescence recovery after photobleaching (FRAP). A circular bilayer spot of $20 \mu \mathrm{m}$ diameter was 
photobleached for five seconds with a $532 \mathrm{~nm}, 100 \mathrm{~mW}$ laser beam. The recovery of the fluorescence intensity within the spot was measured at one second intervals. The corresponding diffusion coefficients were calculated based on the Hankel transform method. ${ }^{[36]}$

Vesicle - Bilayer Adhesion. In order to cover the SLB with SUVs, the SUV solution $(1 \mu \mathrm{g} / \mathrm{ml})$ was injected into the SLB flow chamber for 2 min. During this time the SUVs electrostatically absorb onto the SLB. The injected vesicle concentration was tuned to reach a vesicle coverage on the membrane of approximately 200 vesicle in the complete field of view. Since particle tracking can only be performed when particles are sufficiently separated, a low SUV coverage is required such that there is a significant time interval between two successive encounters with a neighboring SUV. Before measuring the diffusional motions of the SUVs the chamber was flushed with pure buffer for 2 min, eliminating SUVs in bulk obscuring the view of the adhered SUVs.

Fluorescence Microscopy. During the first $600 \mathrm{~s}$ after vesicle adsorption, SUV motion on the SLB was observed using fluorescence microscopy with an inverted Eclipse TE 2000 microscope (Nikon) equipped with a high-pressure mercury lamp, an Apo TIRF 60× oil objective (NA 1.49), and a Luca EMCCD camera $(512 \times 512$ pixel $)$ corresponding to $0.27 \mu \mathrm{m}$ per pixel. Fluorescent images $(137 \times$ $137 \mu \mathrm{m})$ were acquired using $400 \mathrm{~ms}$ exposure time, i.e. a frame rate of $2.5 \mathrm{~Hz}$.

Vesicle Detection. Image analysis was conducted in Matlab R2015b. A vesicle was defined as a group of at least three and no more than 30 connected pixels exceeding an intensity threshold, which is set a few-fold higher than the average noise level. The minimum and maximum SUV pixel sizes ensure the exclusion of noise and clustered SUVs, respectively, from the detected SUVs.

Vesicle Tracking. Vesicle trajectories were constructed by piecing together the SUV positions in consecutive frames, where the separation between consecutive positions should be less than five pixels. Overlapping trajectories (within five pixels) were terminated. Trajectories shorter than ten time steps, or with a diffusivity smaller than $1 \%$ of the mean diffusivity were ignored. Typically a 
vesicle could not be tracked for much longer than $2 \mathrm{~s}$, since this is the typical time between close encounters with neighboring vesicles, at which the vesicle can no longer be distinguished from its neighbor, and the tracking of the vesicle is terminated.

\section{Supporting Information}

Supporting Information is available from the Wiley Online Library or from the author.

\section{Acknowledgements}

This work was supported by the National Research Foundation (NRF-NRFF2011-01). Seyed R. Tabaei and Jurriaan J. J. Gillissen contributed equally to this work.

[1] Singer, S. J.; Nicolson, G. L., Science 1972, 175 (23), 720-31.

[2] Vereb, G.; Szöllősi, J.; Matko, J.; Nagy, P.; Farkas, T.; Vigh, L.; Matyus, L.; Waldmann, T.; Damjanovich, S., Proc. Natl. Acad. Sci. USA 2003, 100 (14), 8053-8058.

[3] Hartman, N. C.; Nye, J. A.; Groves, J. T., Proc. Natl. Acad. Sci. USA 2009, 106 (31), 1272912734.

[4] Salaita, K.; Nair, P. M.; Petit, R. S.; Neve, R. M.; Das, D.; Gray, J. W.; Groves, J. T., science 2010, 327 (5971), 1380-1385.

[5] Stabley, D.; Retterer, S.; Marshall, S.; Salaita, K., Integr Biol-Uk 2013, 5 (4), 659-668.

[6] Zhou, Y.; Mao, H.; Joddar, B.; Umeki, N.; Sako, Y.; Wada, K.-I.; Nishioka, C.; Takahashi, E.; Wang, Y.; Ito, Y., Sci. Rep. 2015, 5.

[7] Evans, E.; Sackmann, E., J. Fluid Mech. 1988, 194, 553-561.

[8] Peters, R.; Cherry, R. J., Proc. Natl. Acad. Sci. USA 1982, 79 (14), 4317-4321.

[9] Merkel, R.; Sackmann, E.; Evans, E., J. Phys. 1989, 50 (12), 1535-1555.

[10] Jönsson, P.; Beech, J. P.; Tegenfeldt, J. O.; Höök, F., Langmuir 2009, 25 (11), 6279-6286.

[11] Datta, A.; Pal, S. K.; Mandal, D.; Bhattacharyya, K., J. Phys. Chem. B 1998, 102 (31), 61146117.

[12] Weiß, K.; Neef, A.; Van, Q.; Kramer, S.; Gregor, I.; Enderlein, J., Biophys. J. 2013, 105 (2), 455-462.

[13] Cicuta, P.; Keller, S. L.; Veatch, S. L., J. Phys. Chem. B 2007, 111 (13), 3328-3331.

[14] Camley, B. A.; Esposito, C.; Baumgart, T.; Brown, F. L., Biophys. J. 2010, 99 (6), L44-L46.

[15] Petrov, E. P.; Petrosyan, R.; Schwille, P., Soft Matter 2012, 8 (29), $7552-7555$.

[16] Yoshina-Ishii, C.; Chan, Y.-H. M.; Johnson, J. M.; Kung, L. A.; Lenz, P.; Boxer, S. G., Langmuir 2006, 22 (13), 5682-5689.

[17] Dimova, R.; Dietrich, C.; Hadjiisky, A.; Danov, K.; Pouligny, B., EPJ B 1999, 12 (4), 589598.

[18] Lee, G. M.; Ishihara, A.; Jacobson, K. A., Proc. Natl. Acad. Sci. USA 1991, 88 (14), 62746278.

[19] Hormel, T. T.; Kurihara, S. Q.; Brennan, M. K.; Wozniak, M. C.; Parthasarathy, R., Phys. 
Rev. Lett. 2014, 112 (18), 188101.

[20] Saffman, P.; Delbrück, M., Proc. Natl. Acad. Sci. USA 1975, 72 (8), 3111-3113.

[21] Tanaka, M.; Sackmann, E., Nature 2005, 437, 656-663.

[22] Ariga, K., Chem. Rec 2004, 3 (6), 297-307. DOI 10.1002/tcr.10071.

[23] Edward T. Castellana; Cremer, P. S., Surf. Sci. Rep. 2006, 61, 429-444.

[24] Evans, E.; Yeung, A., Chem Phys Lipids 1994, 73 (1), 39-56.

[25] Raphael, R. M.; Waugh, R. E., Biophys. J. 1996, 71 (3), 1374.

[26] Chizmadzhev, Y. A.; Kumenko, D. A.; Kuzmin, P. I.; Chernomordik, L. V.; Zimmerberg, J.; Cohen, F. S., Biophys. J. 1999, 76 (6), 2951-2965.

[27] Pfeiffer, W.; König, S.; Legrand, J.; Bayerl, T.; Richter, D.; Sackmann, E., EPL (Europhysics Letters) 1993, 23 (6), 457.

[28] Pott, T.; Méléard, P., EPL (Europhysics Letters) 2002, 59 (1), 87.

[29] Fournier, J.-B.; Khalifat, N.; Puff, N.; Angelova, M., Phys. Rev. Lett. 2009, 102 (1), 018102.

[30] Kalb, E.; Frey, S.; Tamm, L. K., Biochim. Biophys. Acta 1992, 1103 (2), 307-16.

[31] Tamm, L. K.; McConnell, H. M., Biophys. J. 1985, 47 (1), 105-113.

[32] Köchy, T.; Bayerl, T. M., Phys. Rev. E 1993, 47 (3), 2109-2116.

[33] Tabaei, S. R.; Jackman, J. A.; Kim, S.-O.; Liedberg, B.; Knoll, W.; Parikh, A. N.; Cho, N.J., Langmuir 2014, 30 (44), 13345-13352.

[34] Vaz, W. L.; Criado, M.; Madeira, V. M.; Schoellmann, G.; Jovin, T. M., Biochemistry 1982, 21 (22), 5608-5612.

[35] Filipe, V.; Hawe, A.; Jiskoot, W., Pharm. Res. 2010, 27 (5), 796-810.

[36] Jonsson, P.; Jonsson, M. P.; Tegenfeldt, J. O.; Höök, F., Biophys. J. 2008, 95 (11), 53345348. 